\title{
Equal opportunity equivalence in land division
}

\author{
Antonio Nicolò • Andrés Perea y Monsuwe • \\ Paolo Roberti
}

Received: 19 November 2010 / Accepted: 25 May 2011 / Published online: 8 June 2011 C The Author(s) 2011. This article is published with open access at SpringerLink.com

\begin{abstract}
In this paper we look for a solution to a land division problem that could be applied to different types of disputes when the arbitrator has a very limited information about the agents' preferences. The solution must be fair and efficient under the constraint of the limited information available to the arbitrator. To this scope, we propose to use the concept of equal-opportunity equivalence defined by Thomson (Soc Choice Welfare 11:137-156 1994). We prove the existence of an efficient and equal opportunity equivalent allocation for a land division problem and we present a simple procedure to implement a rule that selects such allocation at each preference profile.
\end{abstract}

Keywords Egalitarism · Equity · Fair division · Land division · Implementation

JEL Classification D63 (equity, justice, inequality) · C70 (game theory general) · D30 (distribution general)

We are grateful to an anonymous referee for thoughtful comments that greatly improved the paper. We have also received very useful suggestions from Paolo Dai Pra.

\footnotetext{
A. Nicolò $(\varangle) \cdot$ P. Roberti

Department of Economics, Università degli Studi di Padova, via del Santo 33, 35123 Padua, Italy

e-mail: antonio.nicolo@unipd.it

P. Roberti

e-mail: paolo.roberti@studenti.unipd.it

A. Perea y Monsuwe

Department of Quantitative Economics and EpiCenter,

Maastricht University, P. O. Box 616, 6200 MD Maastricht,

The Netherlands

e-mail: a.perea@maastrichtuniversity.nl
} 


\section{Introduction}

There are several situations where the solution of a land division problem cannot be found using instruments like prices or monetary compensations. This may be due to liquidity constraints; or to the psychological difficulty of bringing a dispute down to monetary evaluations; or the division of the land has to be in kind under the provisions of the law. According to the common law, co-owners of land (or of any other assets) in case of inharmonious association have the right to partition in kind (see Miceli and Sirmans 2000). In case the partition of the land causes excessive fragmentation, a second remedy is the sale of the undivided parcel with division of the proceeds in proportion to each owner's share. This second remedy, however, is considered an extrema ratio that courts should implement only in case there is a strong evidence that land division is inefficient. ${ }^{1}$ In absence of scale economies, division in kind is de facto the only remedy according to the common law. Division in kind is not problematic if the land (or the asset) to be divided is homogeneous, but even if the land is homogeneous for the market value, it could be heterogeneous with respect to owners' preferences: owners' evaluation may depend, in fact, on sentimental considerations, or on private information about the land (for instance, the presence of natural resources in some parcels). If according to owners' preferences the good is not homogeneous, a division in kind can be inefficient, and therefore courts have to try to elicit private informations from the parties.

The case of two countries disputing over their border has similar features. Often, the only feasible remedy is the division in kind. The territory object of the dispute is rarely homogeneous, since many characteristics are relevant to determine the preference of each country: the presence of ethnic groups, of natural resources, geographic characteristics (access to the sea for instance), or strategic and military considerations, etc.. Also in this case a land division may be not efficient and, moreover, it is far from being obvious which normative properties can be called for.

The solution we are looking for and the mechanism to implement it, should hold for a large class of problems. The solution should be applied to solve disputes between co-owners in case of division of land, as also between countries. The solution must be fair and efficient under the constraint of the limited information available to any external party called to settle the dispute (the arbitrator). We assume, in fact, that the arbitrator has not much information about the litigants' preferences, while parties have complete information about each other. Namely the only information available to the arbitrator is that the value of the parcel an agent receives is positively affected by its size; that is, a larger parcel it is always preferred to a smaller one that is contained by it (set-inclusion property). This informational framework fits many real world situations. A judge who has to partition a piece of land between two co-owners, two heirs or a divorcing couple, hardly knows parties' preferences, while it is very likely that each party knows the preferences of the other party over the good to be divided. Similarly, an international organization, like United Nations, which tries to solve a

\footnotetext{
1 "Physical division does not compel a person to sell his property against his will. which, it has been said, should not be done except in case of imperious necessity" Miceli and Sirmans (2000) p.794 quoting the court of the case of Trowbridge v. Donner (1950).
} 
dispute over a territory in the border of two countries cannot claim to know which are the preferences of each country, even if they are common information.

It is important to note that set-inclusion property does not mean that in general any parcel of larger size is preferred to a smaller parcel, since as we pointed out, the land to be divided is heterogenous. However, if preferences satisfy this property, then the following easily follows. For any size $\mu$, consider the preferred parcel of that size by agent $i$, and call it a best parcel of size $\mu$ for agent $i$ (which of course is not necessarily unique). For each agent, his best parcel of size $\mu$ is preferred to his best parcel of size $\mu^{\prime}$ if and only if $\mu \geq \mu^{\prime}$.

As regards the properties that the solution has to satisfy, both efficiency and fairness appear as necessary requirements. An inefficient solution can be renegotiated between the parties: any property of an inefficient solution is not necessarily preserved after renegotiation, and therefore to ask for normative properties without requiring efficiency turns to be a vacuous exercise. In the fair division literature, there are two main ordinal concepts of distributive justice. ${ }^{2}$ The first is the envy-free principle which states that each party should (weakly) prefers its share to anyone else's. This was proposed by Gamow and Stern (1958), but became known in the economics literature after Foley (1967). Efficient and envy-free allocations are ex post stable because no one desires to exchange what he received with anyone else's share. However, there may be many efficient envy-free allocations, and individuals may dispute on which one should be selected. The divide-and-choose mechanism under complete information, for instance, selects among all the efficient envy-free allocations the division that maximizes the payoff of the divider so conflict is likely to shift over how the divider is chosen.

An alternative normative concept is the egalitarian equivalent criterion which states that each party should be indifferent between getting her share and some reference bundle, identical for all agents. However, in this case the conflict is likely to shift on which reference bundle should be chosen, as different reference bundles lead to different shares. Pazner and Schmeidler (1978) suggests eluding the problem by focusing only on those reference bundles that are proportional to the total endowment (assuming efficiency, this leads to a unique selection.). It is not immediately obvious how to extend the "Pazner-Schmeidler" rule when the endowment is a single heterogeneous good. LiCalzi and Nicolò (2009) suggests a way of constructing a reference bundle for a heterogeneous infinitely divisible good. Each agent partitions the good in finitely, or countably many, parcels that she considers as homogeneous. The common refinement of all the agents' partitions divides the good in parcels that are homogeneous for each agent. Hence by choosing the reference bundle among those that are proportional to this common refinement, we end up with a reference bundle that is "proportional" to the total endowment as suggested by Pazner and Schmeidler. However, if the heterogeneous good cannot be partitioned in finitely, or countably many, parcels, then the problem of how to choose the reference bundle still arises.

In this paper we propose to overcome the informational constraints and avoid an arbitrary choice of a reference bundle, using the concept of equal-opportunity equivalence defined by Thomson (1994). Thomson (1994) combines the ideas of equal

\footnotetext{
2 See for instance Berliant et al (1992) and Moulin (2004) for a complete overview of modern contributions to the problem of distributive justice in economics.
} 
opportunities and egalitarian-equivalence in the context of economies with private and public goods. In such environments, an allocation is said to be equal-opportunity equivalent relative to a family of choice sets if there exists some reference set of this family such that each agent is indifferent between the allocation and his best alternative in this reference set. Since here the only commonly known characteristic is the set-monotonic inclusion property of the preference domain, and the size is the unique commonly observable (verifiable) characteristic of any set, we define the concept of equal-opportunity equivalence with respect to a family of sets with the same size. Our solution implies that each agent receives a parcel of the land who makes him indifferent with respect to his best parcel of a given size $\mu$, where the size of the reference set must be the same for both agents. The last question to solve is which size $\mu$ should be considered. Efficiency requires to choose as reference set the largest size $\mu^{*}$ such that both agents are indifferent between the parcel they receive and their best parcel of that size $\mu^{*}$.

In the rest of the paper we first prove the existence of an efficient and equal opportunity equivalent allocation for our problem and we propose a simple procedure to implement a rule that selects such allocation at each preference profile under the assumption that agents have complete information about their preferences. The mechanism is the same used in Nicolò and Perea (2005) that generalizes a mechanism suggested in Crawford (1979) and ameliorated in Demange (1984).

\section{The Model}

The problem we consider is how to divide a piece of land between two agents. Let $X \subseteq \mathbb{R}^{n}$, represent a piece of land which is bounded, connected and Lebesguemeasurable. The set $X$ is the total piece of land to be divided among the two agents. Let $L(X)$ be the set of all Lebesgue-measurable subsets of $X$. By $\mu$ we denote the Lebesgue measure on $\mathbb{R}^{n}$. The utility function $u_{i}$ of agent $i, i=1,2$, is assumed to be a measure on $L(X)$ that is absolutely continuous w.r.t. $\mu$. That is, if $\mu(A)=0$ for some $A \in L(X)$, then also $u_{i}(A)=0$. The number $u_{i}(A)$ represents the utility that agent $i$ assigns to the piece of land $A$. Since $u_{i}$ is absolutely continuous w.r.t. $\mu$ we know by the Radon-Nykodym theorem that there exists a non-negative function $v_{i}: X \rightarrow \mathbb{R}$ such that

$$
u_{i}(A)=\int_{A} v_{i} d \mu
$$

for all $A \in L(X)$.

Definition 2.1 A land division problem is a tuple $P=\left(X, u_{1}, u_{2}\right)$ where $X$ is a connected and Lebesgue measurable subset of $\mathbb{R}^{n}$, and $u_{1}, u_{2}$ are measures on $L(X)$ that are absolutely continuous w.r.t. the Lebesgue measure.

For a given land division problem $P=\left(X, u_{1}, u_{2}\right)$, a feasible land division is a pair $\left(A_{1}, A_{2}\right)$ of subsets such that $A_{2}=X \backslash A_{1}$ and both $A_{1}$ and $A_{2}$ belong to $L(X)$. 
Definition 2.2 A land division rule is a function $D$ that assigns to every land division problem $P$ a feasible land division $D(P)$.

We shall focus on land division rules satisfying two properties: efficiency and (a specific form of) equal opportunity equivalence. Efficiency is defined in the usual sense, that is, a feasible land division $\left(A_{1}, A_{2}\right)$ for $P=\left(X, u_{1}, u_{2}\right)$ is efficient if there is no other feasible division $\left(B_{1}, B_{2}\right)$ for which $u_{i}\left(B_{i}\right)>u_{i}\left(A_{i}\right)$ and $u_{j}\left(B_{j}\right) \geq u_{j}\left(A_{j}\right)$ for $i, j=1,2$ and $i \neq j$. Equal opportunity equivalence states that there should be some number $\lambda>0$ such that both agents are indifferent between the part assigned to them and their most preferred piece of land of size $\lambda$.

Definition 2.3 For a given land division problem $P=\left(X, u_{1}, u_{2}\right)$, a feasible land division $\left(A_{1}, A_{2}\right)$ is equal opportunity equivalent if there is some $\lambda>0$ such that

$$
\begin{aligned}
& u_{1}\left(A_{1}\right)=\max \left\{u_{1}(A) \mid A \in L(X) \text { and } \mu(A)=\lambda\right\} \text { and } \\
& u_{2}\left(A_{2}\right)=\max \left\{u_{2}(A) \mid A \in L(X) \text { and } \mu(A)=\lambda\right\} .
\end{aligned}
$$

A land division rule is said to be efficient (equal opportunity equivalent) if its assigns to every land division problem a feasible land division which is efficient (equal opportunity equivalent).

\section{Efficient and equal opportunity equivalent rules}

In this section we prove that there exist land division rules which are both efficient and equal opportunity equivalent. For the proof of the following theorem we need first the following lemmas:

Lemma 1 For every $R \leq u_{i}(X), r \in[0, R]$ and for every measurable $B \subset X$ such that $u_{i}(B)=R$ there exists a measurable subset $A \subset B$ such that $u_{i}(A)=r_{i}$, where $i=1,2$.

Proof Given the absolute continuity of $u_{i}$ we can apply Theorem 1 in Dubins and Spanier (1961). The convexity of the range $u_{i}(C), C \subset B$, guarantees that exists $A \subset B$ such that $u_{i}(A)=r_{i}$.

Lemma 2 If $u_{j}(\tilde{A} \backslash A)=0$ for every $A \subset \tilde{A}$ such that $u_{i}(A)=r$ we have $u_{j}(\tilde{A})=0$, where $\tilde{A} \subset X, r<u_{i}(\tilde{A})$ and $i, j \in\{1,2\}, i \neq j$.

Proof Let us suppose by contradiction that $u_{j}(\tilde{A}) \neq 0$. That means that there is a set $A \subset \tilde{A}$ such that the Radon-Nikodym derivative $f_{j}$ of $u_{j}$ is larger than 0 almost everywhere on $A$. There are three possibilities: $u_{i}(\tilde{A} \backslash A)$ can be larger, equal or less than $r$. If it is equal to $r$ then we have found a contradiction, because $u_{j}(A)>0$. If $u_{i}(\tilde{A} \backslash A)>r$, using Lemma 1 we can find a set $\bar{A} \subset \tilde{A} \backslash A$ such that $u_{i}(\bar{A})=r$ and $u_{j}(\tilde{A} \backslash \bar{A})>0$, contradicting the assumption. If $u_{i}(\tilde{A} \backslash A)<r$ for Lemma 1 we can find a set $B \subset A$ such that $u_{i}(B)=r-u_{i}(\tilde{A} \backslash A)$, then defining $C:=B \cup(\tilde{A} \backslash A)$ we have that $u_{i}(C)=r$, and $u_{j}(A \backslash B)>0$, which implies $u_{j}(\tilde{A} \backslash C)>0$. 


\section{Lemma 3 The function}

$$
f(\lambda):=\max \left\{u_{i}(A): \mu(A)=\lambda, A \in L(X)\right\}
$$

exists for every $0 \leq \lambda \leq \mu(X)$.

Proof The $\max \left\{u_{i}(A): \mu(A)=\lambda, A \in L(X)\right\}$ can be transformed into $\max \left\{u_{i}(A)\right.$ : $\left.\mu\left(A^{C}\right)=\mu(X)-\lambda, A \in L(X)\right\}$. From the compactness theorem of Dubins and Spanier (1961) we know that the range of the vector $\left(u_{i}(A), \mu\left(A^{C}\right)\right)$ as $A$ varies in $L(X)$ is compact. The vertical section of this range is compact as well so $f(\lambda)$ exists.

Theorem 3.1 (Existence) Let $P=\left(X, u_{1}, u_{2}\right)$ be a land division problem. Then, there exists a feasible land division $\left(A_{1}, A_{2}\right)$ which is both efficient and equal opportunity equivalent.

Proof We first need some notation. Let $L_{1}^{*}(X)$ be the collection of those subsets $A \in L(X)$ such that $u_{1}(A) \geq u_{1}(B)$ for all $B \in L(X)$ with $\mu(B)=\mu(A)$. Let $R_{1}:=u_{1}(X)$ and $R_{2}:=u_{2}(X)$. We define functions $U_{2}, \hat{U}_{2}:\left[0, R_{1}\right] \rightarrow\left[0, R_{2}\right]$ by

$$
\begin{aligned}
U_{2}\left(r_{1}\right):=\max \left\{u_{2}\left(A_{2}\right) \mid\right. & A_{2} \in L(X), \exists A_{1} \in L(X) \text { such that } \\
& \left.u_{1}\left(A_{1}\right)=r_{1} \text { and } A_{2}=X \backslash A_{1}\right\},
\end{aligned}
$$

and

$$
\begin{aligned}
& \hat{U}_{2}\left(r_{1}\right):=\max \left\{u_{2}\left(B_{2}\right) \mid B_{2} \in L(X), \exists B_{1} \in L_{1}^{*}(X)\right. \text { such that } \\
& \left.u_{1}\left(B_{1}\right)=r_{1} \text {, and } \mu\left(B_{2}\right)=\mu\left(B_{1}\right)\right\} \text {. }
\end{aligned}
$$

In the same fashion we define $U_{1}\left(r_{2}\right)$ and $\hat{U}_{1}\left(r_{2}\right)$.

First we need prove that:

1. The functions $\hat{U}_{1}$ and $\hat{U}_{2}, U_{1}$ and $U_{2}$ exist,

2. $\hat{U}_{2}(0) \leq U_{2}(0)=R_{2}$,

3. $U_{2}\left(R_{1}\right) \leq \hat{U}_{2}\left(R_{1}\right)=R_{2}$,

4. $U_{2}$ and $U_{1}$ are weakly decreasing, $\hat{U}_{2}$ and $\hat{U}_{1}$ are weakly increasing (monotonicity property),

5. the functions $U_{2}$ and $\hat{U}_{2}$ are continuous in $r_{1}$,

6. $U_{1}$ is the inverse function of $U_{2}$, and $\hat{U}_{1}$ is the inverse function of $\hat{U}_{2}$, namely $U_{2}\left(r_{1}\right)=U_{1}^{-1}\left(r_{1}\right)$ and $\hat{U}_{2}\left(r_{1}\right)=\hat{U}_{1}^{-1}\left(r_{1}\right)$.

- (Existence) To prove the existence of $\hat{U}_{2}$ we need to prove that for every $r_{1} \in$ $\left[0, R_{1}\right]$ there exists a set $B \subset X$ such that $B \in L_{1}^{*}(X)$ and $u_{1}(B)=r_{1}$. This is equivalent to prove that the function $f:[0, \mu(X)] \rightarrow\left[0, u_{1}(X)\right], f(\lambda):=$ $\max \left\{u_{1}(B) \mid \mu(B)=\lambda\right\}$ is continuous. The existence of $f$ is guaranteed by Lemma 3 . 
$f(\lambda)$ is a weakly increasing function. Let us suppose by contradiction that in $\bar{\lambda} f$ is not continuous. Then there is an $r_{1}$ such that $\lim _{\lambda \rightarrow \bar{\lambda}^{-}} f(\lambda)<r_{1}<\lim _{\lambda \rightarrow \bar{\lambda}}+f(\lambda)$. For that $r_{1}$ consider the function $g(x):=\min \left\{\lambda \mid \mu(A)=\lambda, u_{1}(A)=x\right\} . g\left(r_{1}\right)$ cannot be smaller than $\bar{\lambda}$, because it would violate the maximum condition of $f(\lambda)$. Moreover it cannot be larger than $\bar{\lambda}$, because there would be a couple $(\lambda, f(\lambda))$ such that $\lambda<g\left(r_{1}\right)$ and $f(\lambda)>r_{1}$. If we call $A_{1}$ the set generated by $f(\lambda)$, for Lemma 1 there would be an $\tilde{A}_{1} \subset A_{1}$ such that $u_{1}\left(\tilde{A}_{1}\right)=r_{1}$ and $\tilde{\lambda}=\mu\left(\tilde{A}_{1}\right)<g\left(r_{1}\right)$ which violates the definition of $g$. So $g\left(r_{1}\right)=\bar{\lambda}$, for every $r_{1}$ that respects the inequality. Then $g(x)$ would be constant on $\left[\lim _{\lambda \rightarrow \bar{\lambda}}-f(\lambda), \lim _{\lambda \rightarrow \bar{\lambda}}+f(\lambda)\right]$. This is not possible because $g\left(\lim _{\lambda \rightarrow \bar{\lambda}}+f(\lambda)\right)$ generates a set $A$ that, for Lemma 1, strictly contains an $\hat{A}$ such that $u_{1}(\hat{A})=\lim _{\lambda \rightarrow \bar{\lambda}}-f(\lambda)$. So $\mu(\hat{A})<\bar{\lambda}$, violating the definition of $g$. The existence of the maximum in $\hat{U}_{2}$ is given by Lemma 3. The existence of $U_{2}$ is guaranteed by the compactness theorem in Dubins and Spanier (1961), using the same reasoning of the proof of Lemma 3. The proof of existence of $\hat{U}_{1}$ and $U_{1}$ can be done in the same way.

- The second and the third points are straightforward.

- (Monotonicity) If $U_{2}$ were not decreasing there would be $\tilde{r}_{1}$ and $\hat{r}_{1}$ such that $\tilde{r}_{1}<\hat{r}_{1}$ and $U_{2}\left(\tilde{r}_{1}\right)<U_{2}\left(\hat{r}_{1}\right)$. Defining $\left(\hat{A}_{1}, \hat{A}_{2}\right)$ the partition generated by $U_{2}\left(\hat{r}_{1}\right)$ and $\left(\tilde{A}_{1}, \tilde{A}_{2}\right)$ the partition generated by $U_{2}\left(\tilde{r}_{1}\right)$ for Lemma 1 there must be an $A_{1} \subset \hat{A}_{1}$ such that $u_{1}\left(A_{1}\right)=\tilde{r}_{1}$ and, using the set inclusion property, either $u_{2}\left(X \backslash A_{1}\right)>u_{2}\left(\hat{A}_{2}\right)$, so $u_{2}\left(\tilde{A}_{2}\right)$ cannot be a maximum or $u_{2}\left(X \backslash A_{1}\right)=u_{2}\left(\hat{A}_{2}\right)$. If there is no $A_{1}$ such that the strict inequality is satisfied then for Lemma 2 $u_{2}\left(\hat{A}_{1}\right)=0$ and $U_{2}\left(\hat{r}_{1}\right)=R_{2}$ and it is constant in $\left[0, \hat{r}_{1}\right]$.

Similarly we can prove the monotonicity property of $U_{1}$.

To prove that $\hat{U}_{2}$ is weakly increasing we take $\tilde{r}_{1}$ and $\hat{r}_{1}$ such that $\tilde{r}_{1}<\hat{r}_{1} \cdot \hat{U}_{2}\left(\tilde{r}_{1}\right)$ generates $\left(\tilde{B}_{1}, \tilde{B}_{2}\right)$ such that $u_{1}\left(\tilde{B}_{1}\right)=\tilde{r}_{1}, u_{2}\left(\tilde{B}_{2}\right)=\hat{U}_{2}\left(\tilde{r}_{1}\right)$ and $\mu\left(\tilde{B}_{1}\right)=\mu\left(\tilde{B}_{2}\right)$. Then for any set $B_{1} \in L(X)$ such that $u_{1}\left(B_{1}\right)=\hat{r}_{1}$ we will have $\mu\left(B_{1}\right) \geq \mu\left(\tilde{B}_{1}\right)$, otherwise $\tilde{B}_{1} \notin L_{1}^{*}(X)$. Then $\mu\left(B_{1}\right) \geq \mu\left(\tilde{B}_{2}\right)$ and any set $B_{2}$ such that $\tilde{B}_{2} \subset B_{2}$ and $\mu\left(B_{2}\right)=\mu\left(B_{1}\right)$ will satisfy the following inequality: $u_{2}\left(B_{2}\right) \geq u_{2}\left(\tilde{B}_{2}\right)$, proving that $\hat{U}_{2}$ is weakly increasing. We can be more specific and state that $\hat{U}_{2}$ can be constant only if it is equal to $R_{2}$. In fact if $\hat{U}_{2}$ is constant on $\left[\bar{r}_{1}, \hat{r}_{1}\right]$ then calling $\hat{A}_{1}$ and $\bar{A}_{1}$ the maximal sets where $\hat{r}_{1}$ and $\bar{r}_{1}$ are assumed, it must be $\mu\left(\hat{A}_{1}\right)>\mu\left(\bar{A}_{1}\right)$. The same must hold for the maximal sets $\hat{A}_{2}$ and $\bar{A}_{2}: \mu\left(\hat{A}_{1}\right)=\mu\left(\hat{A}_{2}\right)>\mu\left(\bar{A}_{2}\right)=\mu\left(\bar{A}_{1}\right)$. Then if $u_{2}\left(\hat{A}_{2}\right)=u_{2}\left(\bar{A}_{2}\right)$ the Radon-Nikodym derivative $f_{2}$ must be zero almost everywhere on $X \backslash \bar{A}_{2}$ and $\hat{U}_{2}\left(\bar{r}_{1}\right)=R_{2}$.

In the same fashion we can prove that $\hat{U}_{1}$ is weakly increasing.

- (Continuity) To prove the continuity of $U_{2}$ let us do it by contradiction: suppose that in $\tilde{r}_{1} U_{2}$ is not a continuous. For the monotonicity property it must be a jump discontinuity and moreover $\lim _{\tilde{r}_{1}^{-}} U_{2}\left(r_{1}\right)>\lim _{\tilde{r}_{1}^{+}} U_{2}\left(r_{1}\right)$. Consider $U_{1}\left(\tilde{r}_{2}\right)$, where we take $\lim _{\tilde{r}_{1}^{+}} U_{2}\left(r_{1}\right)<\tilde{r}_{2}<\lim _{\tilde{r}_{1}^{-}} U_{2}\left(r_{1}\right)$. Then $U_{1}\left(\tilde{r}_{2}\right)$ cannot be larger than $\tilde{r}_{1}$, otherwise $U_{2}\left(U_{1}\left(\tilde{r}_{2}\right)\right)<\tilde{r}_{2}$ and would not be a maximum. Moreover $U_{1}\left(\tilde{r}_{2}\right)$ cannot be smaller than $\tilde{r}_{1}$. Indeed for the monotonicity property there would be a couple $\left(r_{1}, U_{2}\left(r_{1}\right)\right)$ such that $U_{2}\left(r_{1}\right)>\tilde{r}_{2}$ and $r_{1}>U_{1}\left(\tilde{r}_{2}\right)$. This is not possible because, calling $\left(\bar{A}_{1}, \bar{A}_{2}\right)$ the partition generated by $U_{2}\left(r_{1}\right)$, for Lemma 1 there would be an $A_{2} \subset \bar{A}_{2}$ such that $u_{2}\left(A_{2}\right)=\tilde{r}_{2}$ and $u_{1}\left(X \backslash A_{2}\right) \geq r_{1}$, and $U_{1}\left(\tilde{r}_{2}\right)$ would not 
be a maximum. So $U_{1}\left(\tilde{r}_{2}\right)=\tilde{r}_{1}$. Being $\tilde{r}_{1}$ any real number between $\lim _{\tilde{r}_{1}^{+}} U_{2}\left(r_{1}\right)$ and $\lim _{\tilde{r}_{1}^{-}} U_{2}\left(r_{1}\right) U_{1}$ must be constant in $\left[\lim _{\tilde{r}_{1}^{+}} U_{2}\left(r_{1}\right), \lim _{\tilde{r}_{1}^{-}} U_{2}\left(r_{1}\right)\right]$. This is not possible because $U_{1}$ and $U_{2}$ can be constant only when they are respectively equal to $R_{1}$ and $R_{2}$, where there would be no matter of discontinuity. Indeed let's call $\left(\hat{A}_{1}, \hat{A}_{2}\right)$ the partition generated by $U_{1}\left(\lim _{\tilde{r}_{1}^{-}} U_{2}\left(r_{1}\right)\right)$. Then for all the feasible sets $A_{2} \subset \hat{A}_{2}$ such that $u_{2}\left(A_{2}\right)=\lim _{\tilde{r}_{1}^{+}} U_{2}\left(r_{1}\right)$ it must be that $u_{1}\left(\hat{A}_{2} \backslash A_{2}\right)=0$. Then for Lemma $2 u_{1}\left(\hat{A}_{2}\right)=0$, which implies $u_{1}\left(\hat{A}_{1}\right)=R_{1}$ and $U_{1}\left(\lim _{\tilde{r}_{1}^{-}} U_{2}\left(r_{1}\right)\right)=R_{1}$. Then it must be $\lim _{\tilde{r}_{1}^{-}} U_{2}\left(r_{1}\right)=\lim _{\tilde{r}_{1}^{+}} U_{2}\left(r_{1}\right)$ so $U_{2}$ must be continuous. We can prove in the same fashion the continuity of $U_{1}, \hat{U}_{2}, \hat{U}_{1}$.

- (Inverse functions) Let us suppose by contradiction that in $r_{1} U_{2}\left(r_{1}\right)<U_{1}^{-1}\left(r_{1}\right)$. This is not possible because $U_{2}\left(r_{1}\right)$ should be the maximum of $u_{2}\left(A_{2}\right)$ such that $u_{1}\left(X \backslash A_{2}\right)=r_{1}$ but $U_{1}^{-1}\left(r_{1}\right)$ would generate a partition $\left(\tilde{A}_{1}, \tilde{A}_{2}\right)$ such that $u_{1}\left(\tilde{A}_{1}\right)=r_{1}$ and $u_{2}\left(\tilde{A}_{2}\right)>u_{2}\left(A_{2}\right)$. Instead if $U_{2}\left(r_{1}\right)>U_{1}^{-1}\left(r_{1}\right)$ for the monotonicity property we would have that $r_{1}<U_{2}^{-1}\left(U_{1}^{-1}\left(r_{1}\right)\right)=r_{1}^{*}$, but that would mean that there is partition $\left(\tilde{A}_{1}, \tilde{A}_{2}\right)$ generated by $U_{2}\left(r_{1}^{*}\right)$ such that $u_{2}\left(\tilde{A}_{2}\right)=U_{1}^{-1}\left(r_{1}\right)$ but $u_{1}\left(\tilde{A}_{1}\right)>r_{1}$. This is a violation of the maximum condition of $U_{1}$.

So $U_{2}\left(r_{1}\right)=U_{1}^{-1}\left(r_{1}\right)$. The proof that $\hat{U}_{2}\left(r_{1}\right)=\hat{U}_{1}^{-1}\left(r_{1}\right)$ comes from the definition of the two functions.

By these properties, applying the Bolzano Theorem to the function $\left(U_{2}-\hat{U}_{2}\right)\left(r_{1}\right)$, there must be some $r_{1}^{*}$ such that $U_{2}\left(r_{1}^{*}\right)=\hat{U}_{2}\left(r_{1}^{*}\right)$. Let $r_{2}^{*}:=U_{2}\left(r_{1}^{*}\right)=\hat{U}_{2}\left(r_{1}^{*}\right)$. By definition of $U_{2}$, there are subsets $A_{1}, A_{2} \in L(X)$ such that

1. $u_{1}\left(A_{1}\right)=r_{1}^{*}$ and $u_{2}\left(A_{2}\right)=r_{2}^{*}$,

2. $A_{2}=X \backslash A_{1}$, and

3. given that $U_{2}\left(r_{1}\right)=U_{1}^{-1}\left(r_{1}\right)$ and $\hat{U}_{2}\left(r_{1}\right)=\hat{U}_{1}^{-1}\left(r_{1}\right)$ there is no feasible land division $\left(A_{1}^{\prime}, A_{2}^{\prime}\right)$ with $u_{1}\left(A_{1}^{\prime}\right) \geq r_{1}^{*}$ and $u_{2}\left(A_{2}^{\prime}\right) \geq r_{2}^{*}$ such that $\left(u_{1}\left(A_{1}^{\prime}\right), u_{2}\left(A_{2}^{\prime}\right)\right) \neq$ $\left(r_{1}^{*}, r_{2}^{*}\right)$.

Since the function $U_{2}$ is weakly decreasing and $\hat{U}_{2}$ is weakly increasing it follows from (1), (2) and (3) that $\left(A_{1}, A_{2}\right)$ is a feasible and efficient land division with $u_{1}\left(A_{1}\right)=r_{1}^{*}$ and $u_{2}\left(A_{2}\right)=r_{2}^{*}$.

On the other hand, by definition of $\hat{U}_{2}$ there are subsets $B_{1}, B_{2} \in L(X)$ and a number $\lambda>0$ such that

4. $u_{1}\left(B_{1}\right)=r_{1}^{*}$ and $u_{2}\left(B_{2}\right)=r_{2}^{*}$,

5. $\mu\left(B_{1}\right)=\mu\left(B_{2}\right)=\lambda$,

6. $u_{1}\left(B_{1}\right)=\max \left\{u_{1}(B) \mid B \in L(X)\right.$ and $\left.\mu(B)=\lambda\right\}$, and

7. $u_{2}\left(B_{2}\right)=\max \left\{u_{2}(B) \mid B \in L(X)\right.$ and $\left.\mu(B)=\lambda\right\}$.

Here, (6) follows from the assumption that $B_{1} \in L_{1}^{*}(X)$. By (4)-(7), and our previous insight that $\left(A_{1}, A_{2}\right)$ is a feasible and efficient land division with $u_{1}\left(A_{1}\right)=r_{1}^{*}$ and $u_{2}\left(A_{2}\right)=r_{2}^{*}$, it follows that the feasible land division $\left(A_{1}, A_{2}\right)$ is efficient and equal opportunity equivalent. This completes the proof. 
Theorem 3.2 (Uniqueness) All the efficient and equal opportunity equivalent land divisions $\left(A_{1}, A_{2}\right)$ yield a unique utility pair $\left(u_{1}\left(A_{1}\right), u_{2}\left(A_{2}\right)\right)$ if the Radon-Nikodym derivatives $f_{1}$ and $f_{2}$ respectively of $u_{1}$ and $u_{2}$ are different from zero almost everywhere on $X$.

Proof If the Radon-Nikodym derivatives $f_{1}$ and $f_{2}$ are different from zero a.e. on $X$ the function $U_{2}$ is strictly decreasing and the function $\hat{U}_{2}$ is strictly increasing on $\left[0, R_{1}\right]$. Then they cross just in one point $\left(r_{1}, r_{2}\right)$ which is the unique utility pair that can be obtained by any efficient and equal opportunity equivalent land division.

\section{The mechanism}

In this section we present a simple mechanism to implement an efficient and equal opportunity equivalent allocation when agents have complete information.

Round 1. Agent 1 announces $\lambda \in[0, \mu(X)]$. Agent 2 can take any portion $B_{2}$ such that $\mu\left(B_{2}\right) \leq \lambda$ or propose a land division $\left(A_{1}, A_{2}\right) \in L(X) \times L(X)$. If agent 2 takes $B_{2}$, agent 1 gets $X \backslash B_{2}$. If agent 2 proposes a land division $\left(A_{1}, A_{2}\right) \in L(X) \times L(X)$, then round 2 is played

Round 2. Agent 1 can accept agent 2's proposal and in this case the proposal is implemented, or reject it and take any portion $B_{1}$ with $\mu\left(B_{1}\right) \leq \lambda$. In case of rejection agent 2 gets the portion $X \backslash B_{1}$.

For any $\lambda \in[0, \mu(X)]$ and for any $i=1,2$ let $B_{i}^{\lambda}=\left\{B \in L(X) \mid \arg \max u_{i}(B)\right.$ s.t. $\mu(B) \leq \lambda\}$.

The equilibrium concept used is subgame perfection; for short, we simply speak of "equilibrium".

Theorem 4.1 The mechanism described above has unique equilibrium payoffs, with final allocations that are efficient and equal opportunity equivalent. In every equilibrium allocation, each agent $i$ is indifferent between the parcel he receives and getting $u_{i}\left(B_{i}^{\lambda^{*}}\right)$, where $\lambda^{*}=\max \left\{\lambda\right.$ : there exists $\left(A_{1}, A_{2}\right) \in L(X) \times L(X)$ with $u_{i}\left(A_{i}\right) \geq u_{i}\left(B_{i}^{\lambda}\right)$ for each $\left.i\right\}$.

Proof We proceed by backward induction. Consider first round 2 and suppose that agent 1 has announced $\lambda$ at round 1 and agent 2 has made a proposal $\left(A_{1}, A_{2}\right)$. It is straightforward to note that agent 1 accepts agent 2's proposal if and only if $u_{1}\left(A_{1}\right) \geq u_{1}\left(B_{1}^{\lambda}\right)$, otherwise he takes $B_{1}^{\lambda}$. Consider round 1 . Suppose that agent 1 has announced $\lambda$. We show now that agent 2 proposes the allocation

$$
\left(A_{1}^{\lambda}, A_{2}^{\lambda}\right) \in\left\{\left(A_{1}, A_{2}\right) \in L(X) \times L(X) \mid \arg \max u_{2}(\cdot) \text { s.t. } u_{1}\left(A_{1}\right)=u_{1}\left(B_{1}^{\lambda}\right)\right\}
$$

if and only if $u_{2}\left(A_{2}^{\lambda}\right) \geq u_{2}\left(B_{2}^{\lambda}\right)$; if $u_{2}\left(A_{2}^{\lambda}\right)<u_{2}\left(B_{2}^{\lambda}\right)$ then he takes the portion $B_{2}^{\lambda}$. We have already argued that agent 1 accepts a proposal if and only $u_{1}\left(A_{1}\right) \geq u_{1}\left(B_{1}^{\lambda}\right)$. Since agent 2 can chop off a morsel of agent 1's portion and give it to himself, then if he makes a proposal $\left(A_{1}, A_{2}\right)$ it must be that $u_{1}\left(A_{1}\right) \leq u_{1}\left(B_{1}^{\lambda}\right)$. Hence agent 
2's best proposal satisfies the constraint $u_{1}\left(A_{1}\right)=u_{1}\left(B_{1}^{\lambda}\right)$. Agent 2 makes a proposal only if there exists a proposal which guarantees to him a payoff higher than the payoff he gets by taking the portion $B_{2}^{\lambda}$ (note that any agent 2's proposal that is rejected by agent 1 is payoff equivalent to a (acceptable) proposal $\left(A_{1}, A_{2}\right)=$ $\left.\left(B_{1}^{\lambda}, X \backslash B_{1}^{\lambda}\right)\right)$. Let $\mathbf{A}=\left\{\left(A_{1}^{\lambda}, A_{2}^{\lambda}\right)\right.$ for some $\lambda \in[0, \mu(X)]$ and $\left.u_{2}\left(A_{2}^{\lambda}\right) \geq u_{2}\left(B_{2}^{\lambda}\right)\right\}$ and $A^{\lambda^{*}}=\left(A_{1}^{\lambda^{*}}, A_{2}^{\lambda^{*}}\right) \in \mathbf{A}$ be such that for all $A^{\lambda} \in \mathbf{A}, \lambda \leq \lambda^{*}$. We show as last step of the proof that at round 1 , agent 1 proposes $\lambda^{*}$. Suppose agent 1 proposes $\lambda^{*}$ at round 1 ; agent 2 proposes the allocation $\left(A_{1}^{\lambda^{*}}, A_{2}^{\lambda^{*}}\right)$ and agent 1 accepts. By proposing $\lambda^{*}$ at round 1 agent 1 gets $u_{1}\left(A_{1}^{\lambda^{*}}\right)=u_{1}\left(B_{1}^{\lambda^{*}}\right)$. Suppose agent 1 proposes $\lambda<\lambda^{*}$. For all $\lambda<\lambda^{*}\left(A_{1}^{\lambda}, A_{2}^{\lambda}\right) \in \mathbf{A}$, agent 2 proposes an allocation that satisfies condition (4.1) and agent 1 obtains $u_{1}\left(B_{1}^{\lambda}\right)<u_{1}\left(B_{1}^{\lambda^{*}}\right)$. Suppose, finally, that agent 1 proposes $\lambda>\lambda^{*}$. By definition of $\lambda^{*},\left(A_{1}^{\lambda}, A_{2}^{\lambda}\right) \notin \mathbf{A}$ and $u_{2}\left(A_{2}^{\lambda}\right)<u_{2}\left(B_{2}^{\lambda}\right)$. But then agent 2 takes $B_{2}^{\lambda}$ and agent 1 gets $X \backslash B_{2}^{\lambda}$. Since $\lambda>\lambda^{*}$, then $u_{2}\left(B_{2}^{\lambda}\right)>u_{2}\left(B_{2}^{\lambda^{*}}\right)$. Since by construction $\left(A_{1}^{\lambda^{*}}, A_{2}^{\lambda^{*}}\right)$ is an efficient allocation, it follows that $u_{1}\left(X \backslash B_{2}^{\lambda}\right)<u_{1}\left(A_{1}^{\lambda^{*}}\right)=u_{1}\left(B_{1}^{\lambda^{*}}\right)$.

Open Access This article is distributed under the terms of the Creative Commons Attribution License which permits any use, distribution and reproduction in any medium, provided the original author(s) and source are credited.

\section{References}

Berliant M, Dunz K, Thomson W (1992) On the fair division of a heterogeneous commodity. J Math Econ 21(3):201-216

Crawford VP (1979) A procedure for generating Pareto-efficient egalitarian-equivalent allocations. Econometrica 47:49-60

Demange G (1984) Implementing efficient egalitarian equivalent allocations. Econometrica 52:1167-1177

Foley D (1967) Resource allocation and the public sector. Yale Econ Essays 7:45-98

Gamow G.G., Stern M (1958) Puzzle-math. The Viking Press, New York

LiCalzi M, Nicolò A (2009) Egalitarian equivalent allocations over a single good. Econ Theory 40(1):27-45

Miceli TJ, Sirmans CF (2000) Partition of real estate; or, breaking up is (not) hard to do. J Legal Stud 29:783-796

Moulin H (2004) Fair division and collective welfare, MIT Press, Cambridge

Nicolò A, Perea A (2005) Monotonicity and equal-opportunity equivalence in bargaining. Math Soc Sci 49:221-243

Pazner EA, Schmeidler D (1978) Egalitarian equivalent allocations: a new concept of economic equity. Q J Econ 92:671-687

Thomson W (1994) Notions of equal, or equivalent, opportunities. Soc Choice Welfare 11:137-156

Weller D (1985) Fair division of a measurable space. J Math Econ 14:5-17 